\title{
Heidegger y la noción de verdad en las ciencias ónticas: una reconstrucción de los sentidos de verdad que imperan en las ciencias de lo intramundano*
}

\author{
Heidegger and the notion of truth in the ontic sciences: a \\ rebuilding of the meanings of truth that reign over the sciences \\ of the intra-worldly
}

LUCIANO MASCARÓ**

\begin{abstract}
Resumen: A continuación nos proponemos investigar la filosofía heideggeriana de los años '20 (el así llamado "período de Marburgo") para determinar el sentido de verdad que opera como trasfondo en las labores de las ciencias ónticas de lo intramundano (ciencias naturales y físico-matemáticas). Nuestro recorrido nos mostrará cuatro sentidos fundamentales: La verdad como propiedad de los enunciados; como resultados y consecuencias; como concordancia de la predicción con el experimento, y como certeza. Concluiremos nuestro estudio con la discusión de la posibilidad de subsistencia de la verdad con anterioridad al despliegue de la existencia, lectura que será criticada por medio de la distinción entre verdad y efectividad.

Palabras clave: Heidegger, verdad, ciencia, enunciado, experimento.
\end{abstract}

\begin{abstract}
In this study we attempt to investigate heidegger's philosophy from the 1920's (the so-called "Marburg period") in order to determine de meaning of truth which functions as a background for the activities of the ontic sciences of the intra-worldly (natural and physic-mathematical sciences). Our track will show four fundamental meanings; Truth as a feature of statements; as results and consequences; as concordance between the experiment and the prediction; and as certanty. We will conclude our research whith the discusion of the possibility of truth's subsistence as prior to the existence's unfolding, interpretation that will be critisized by means of the distinction between truth and efectiveness.
\end{abstract}

Keywords: Heidegger, Truth, Science, Statement, Experiment.

Fecha de recepción: 24/06/2015. Fecha de aceptación: 12/11/2015.

* Este trabajo surge del proyecto de investigación Pip-CONICET (2014-2016) N 11220130100505 "Fuerza y significado. La primera articulación significativa de la lógica hermenéutica". Resolución del Directorio de CONICET 5013/2014. Desde el 01.06.2015 hasta el 31.05.2017. Lugar de trabajo: Centro de Estudios Filosóficos "Eugenio Pucciarelli"

** Investigador Postdoctoral en el Consejo Nacional de Investigaciones Científicas y Técnicas (CONICET); Investigador en la Academia Nacional de Ciencias de Buenos Aires - Centro de Estudios Filosóficos (ANCBA-CEF). Email: 1cnmascaro@hotmail.com. Líneas de investigación: Hermenéutica, Fenomenología, Epistemología, Filosofía de la Tecnología, Filosofía contemporánea. Publicaciones recientes: "Las pasiones científicas: en busca del perfil afectivo de la ciencia en la filosofía heideggeriana del período de Marburgo". En Límite: Revista interdisciplinaria de psicología y filosofía, Vol.9, n²9, año 2014, Universidad de Tarapacá, Chile, ed. De la universidad de Tarapacá. ISSN 0718-1361, pp. 92-111. "Notas acerca de la relación teoría-praxis en la filosofía del primer Heidegger" en Artefacto, obra y discurso: Lógica hermenéutica y producción. (Adrián Bertorello y Diego Parente comp.) Ed. Teseo, Buenos Aires: 2014. ISBN 978-987-723-016-1. 


\section{Introducción}

A continuación nos proponemos investigar la filosofía heideggeriana de los años '20 (el así llamado "período de Marburgo") para determinar el sentido de verdad que opera como trasfondo en las labores de las ciencias ónticas de lo intramundano (ciencias naturales y físico-matemáticas). Si bien existen lecciones dedicadas manifiestamente al problema de la verdad, la cuestión específica que nos concierne aparece de forma dispersa en las obras del período, de modo que nuestro trabajo será de reconstrucción y articulación de las temáticas. En nuestro trayecto tomaremos como presupuesto el análisis existencial de la verdad en tanto apertura y descubrimiento, y avanzaremos hacia la caracterización de la verdad como una propiedad de los enunciados apofánticos. Concentrándonos en esta interpretación derivada de la verdad, la cual da origen a la difundida concepción que hace de la ciencia un entramado de proposiciones fundamentadas (el así llamado "concepto lógico"1), estudiaremos las interpretaciones que las ciencias naturales hacen de la noción de verdad; nuestro recorrido nos mostrará cuatro sentidos fundamentales: la verdad como propiedad de los enunciados; como resultados y consecuencias; como concordancia de la predicción con el experimento, y como certeza. Concluiremos nuestro estudio con el análisis de la expresión "hay verdad", que parece indicar la existencia de verdades "subsistentes" anteriores al despliegue descubridor de la existencia, a las cuales las leyes científicas deberían plegarse, lectura que será criticada por medio de la distinción entre verdad y efectividad.

Si bien la etapa del pensamiento de Heidegger al que nuestra investigación se cierne es el de las lecciones ofrecidas entre los años 1923 y $1927^{2}$, deberemos hacer frecuente referencia a una obra perteneciente a un período posterior, pero indispensable para nuestra búsqueda debido a la fuerte familiaridad temática: los seminarios de Zollikon

\section{Demarcación preliminar de los sentidos de verdad}

Heidegger se ocupa expresamente del análisis de los sentidos más difundidos del término "verdad" por medio de dos aproximaciones últimamente convergentes: la primera de ellas aparece en Lógica: la pregunta por la verdad (de 1925), y la segunda en Ser y Tiempo. En su lección de 1925, Heidegger expone tres interpretaciones tradicionales (que encarnan tres prejuicios) acerca de esta noción: el primer prejuicio, a saber, que la verdad pertenece a la proposición como a su lugar propio, es puesto en duda por medio de un retorno y reconstrucción de la fuente griega; en este retorno descubrimos que la formulación del perí hermenéias presenta al enunciado apofántico como el tipo de discurso donde acontece el ser verdadero o ser falso, luego, la verdad como fenómeno debe preexistir a la enunciación. El tercer prejuicio, esto es, que el autor de la concepción que hace de la proposición el lugar propio de la verdad sería Aristóteles, se desploma por el mismo análisis de los textos originales. Habiendo, pues, sometido a crítica el primer y el tercer prejuicio, Heidegger se ocupa de analizar el segundo prejuicio: "la verdad consiste en la concordancia entre el pensamiento y la realidad". Este análisis llevará a una reformulación de la idea de adaequatio desde un punto de vista existencial; la concordancia no

1 (Cfr. Heidegger, 2006:373).

2 Específicamente, nos concentraremos en aquellas lecciones donde el problema de la verdad sea tratado de manera más explícita. 
nombrará ya la correspondencia entre el pensamiento y la cosa, sino entre el modo de mostrar del lógos, y el modo de aparecer de los phainómena, siempre ante una existencia que se despliega.

En Ser y Tiempo, Heidegger realiza una exposición de los sentidos cotidianos en los que se comprende el término "verdad", en este análisis encontramos: a) la verdad como un carácter de los enunciados; b) la verdad como una determinada proposición en sí (por ejemplo, una ley científica); c) la verdad como una situación subjetiva de toma de conciencia; o d) verdad como la totalidad de proposiciones que deben ser formuladas para conocer acabadamente un incidente o acontecimiento.

Si lo que nos proponemos es determinar precisamente en qué sentido se dice "verdad" en las ciencias ónticas de lo intramundano (ciencias naturales y físico-matemáticas), observamos desde un comienzo que algunos de los sentidos expuestos no resultarán especialmente relevantes para la realización de nuestra tarea; de aquí en más, los sentidos cotidianos de verdad que se volverán objeto de nuestro análisis, serán los dos primeros, a saber, verdad como calificativo de la proposición, o bien verdad como "una determinada proposición verdadera". Por su parte, el prejuicio al que nos dedicaremos más específicamente será el segundo, a saber, el que interpreta a la verdad como adecuación.

\section{El problema de la verdad en el discurso científico}

A lo largo de las lecciones del período de Marburgo podemos observar que la interpretación más difundida acerca de la estructura de la ciencia es la que hace de ella un entramado de enunciados apofánticos que se vinculan por medio de relaciones de fundamentación; esta concepción es denominada concepto lógico [logische Begriff] de la ciencia. Dado que esta interpretación constituye la lectura dominante por medio de la cual se accede al problema de la ciencia, podemos comenzar a divisar el lugar que ocupará la idea de verdad en las tareas de las disciplinas que tematizan lo intramundano: ellas se guían por la concepción de la verdad como una característica de la proposición enunciativa. Ya Husserl había identificado a la ciencia con un conjunto de contenidos ideales, a cada proposición le correspondería una verdad (Cfr. Husserl, 2006:199). Una efectiva prueba de que el contenido de una ley científica es una idealidad es el carácter universal de su formulación; en efecto, las leyes se refieren a todos los casos posibles a pesar de que ningún observador empírico podría jamás encontrarse ante todos los casos posibles de un fenómeno (Cfr. Heidegger, 2007b:57) una ley científica de este tipo constituye, al fin y al cabo, una suposición [Unterstellung]. La unidad de todas las proposiciones acerca de un mismo ámbito del ser constituiría la estructura total de una ciencia (en conjunción con los actos, artefactos e instituciones que la componen). La ciencia es, según esta postura, una estructura reticular de verdades, cada una de las cuales contiene y expresa una cierta regularidad observable en el mundo (esta situación es especialmente perceptible en las ciencias naturales, donde rige la idea de naturaleza en tanto legalidad, noción tributaria del proyecto matemático ${ }^{3}$ ).

Con todo, la asociación de la ciencia con la concepción de la verdad en tanto verdad de la proposición no parece llegar a afectar las consideraciones del hombre común. En los Seminarios de Zollikon, Heidegger se pregunta de qué manera percibe el "hombre de la calle" la verdad de la ciencia; su respuesta: en los resultados. Efectivamente, para la cotidianidad, para el hombre que no se dedica expresamente a la tarea del descubrimiento temático de

3 (Cfr Heidegger 2006: 378 y 1995b:30 y ss.) 
los entes en sus propiedades, para aquel que no se pregunta por regularidades o fórmulas, la veracidad de la ciencia se ve confirmada por su modo de influir en la vida cotidiana; la ciencia "demuestra" que sus propuestas son válidas por medio de la introducción de nuevos productos en el vivir de la medianía; artefactos, técnicas, trabajos e instituciones que se vuelven parte de la totalidad de entes y procesos que configuran el mundo público (Cfr. Heidegger, 2007b:85). El hombre común puede no haberse preguntado jamás por la ley que rige el movimiento de la máquina a vapor, y sin embargo, ha dejado que su vida quede organizada en torno a trenes y barcos ${ }^{4}$. El hombre de la medianía confía en que debe haber un por qué, un fundamento, una fórmula, una demostración que explica y se coloca por debajo el funcionamiento del nuevo artefacto o, por ejemplo, del nuevo método de sanación, pero esta pregunta no se inmiscuye en el día a día, esta pregunta y su respuesta queda reservada para el científico; el hombre de término medio (el modo en el que todos nos encontramos la mayor parte del tiempo) sólo se deja beneficiar por las capacidades prácticas de la novedad. Para el hombre común, la ciencia debe lidiar con verdades, con valideces, puesto que sus estudios están produciendo efectivamente resultados visibles en el trato cotidiano. Por ello afirma Heidegger: "El hombre de la calle ve la verdad de la física únicamente en su efecto, es decir, en la figura del auto que maneja" (Heidegger, 2007b:85) Aunque la ley no se comprenda ni se cuestione, (incluso, aunque ella no haya sido aún formulada) el hombre cotidiano convive con el hecho de que cada vez es posible viajar más rápido, vivir más tiempo, conversar a mayor distancia ${ }^{5}$. Esta es la concepción cotidiana de la verdad de la ciencia: la verdad se observa en sus resultados, plasmados en artefactos y técnicas que modifican la vida diaria.

Ahora bien, esta concepción de la verdad como efecto o resultado no sólo afecta a la consideración del hombre común, sino que también opera (aunque desde otra perspectiva) en las labores específicas de la ciencia. También para el científico la veracidad de una ley (su carácter efectivamente descriptivo de un fenómeno) queda confirmada en el resultado, en este caso, en el resultado de una experimentación. Es interesante observar cómo la clásica noción de verdad como adaequatio sigue vigente -aunque modificada- en las investigaciones de la ciencia actual. Heidegger se encarga de ilustrar esta derivación en su escrito de juventud El concepto de tiempo en la ciencia histórica; allí se señala que una diferencia fundamental entre la ciencia antigua y la moderna es el modo de proponer las explicaciones (teorías): en la ciencia antigua (y también medieval), el desarrollo de una explicación comienza por la observación de una serie de fenómenos de un mismo tipo ${ }^{6}$, para luego establecer los caracteres comunes a todos ellos, lo cual llevará a la determinación de la esencia del fenómeno (no necesariamente expresada en una ley, y menos aún una ley aritmética); en el caso de la ciencia moderna (cuyo proyecto matemático rige aún hoy) el proceso de formulación de regularidades funciona de manera diferente, tomando como ejemplo a Galileo, Heidegger afirma que el científico (natural) moderno comienza por la propuesta de un supuesto general

4 Paradójicamente, la ley que explica el funcionamiento de la máquina a vapor, -la segunda ley de la termodinámica- (1824-1860) fue formulada 50 años después de la invención y desarrollo del artefacto (1774). Este dato no es de común conocimiento, y por ello no defrauda la confianza del hombre común en los resultados de la ciencia.

5 Desde luego, los efectos del desarrollo de las teorías no sólo redundan en el beneficio del hombre, sino también en su perjuicio, por ejemplo, el que adviene como consecuencia del avance tecnológico en el terreno militar.

6 ¿Qué es lo que caracteriza de antemano a los fenómenos como pertenecientes a "un mismo tipo"? respondemos: El proyecto previo de la constitución de una región del ser. La ciencia en tanto apertura de un a priori (Cfr. Heidegger, 2006:378). 
(hipótesis), expresada en una fórmula matemática; esta ecuación luego se pone a prueba por medio de una serie de experiencias diseñadas ad hoc; si la hipótesis es confirmada por las observaciones, ella gana en validez, es decir, en veracidad, en credibilidad. "Así, pues, es válido el anterior supuesto a partir del cual se obtuvo de forma puramente deductiva la ley que mas tarde se confirmó experimentalmente." (Heidegger, 2009:20-21).

"En este nuevo método encontramos, por tanto, dos rasgos peculiares: 1) Se establece una hipótesis que hace inteligibles los fenómenos de un determinado ámbito, en este caso, los fenómenos del movimiento. 2) La hipótesis no afirma una cualidad oculta como la causa que explica los fenómenos, sino que contiene relaciones matemáticamente comprensibles -es decir, mensurables- entre los momentos idealmente concebidos del fenómeno" (Heidegger, 2009:21)

Hace un momento habíamos propuesto que en la ciencia moderna, la idea de adaequatio sigue vigente, aunque modificada; ahora precisamos aquella afirmación: en la ciencia óntica (fundamentalmente la natural), la concordancia no será ya la que se establece entre el pensamiento y la realidad, sino entre la explicación (hipótesis, en tanto elemento central de la teoría) formulada de antemano y el resultado del experimento que se diseña para ponerla a prueba. Esta situación demuestra que también para el científico (no sólo para el hombre común), la veracidad de una teoría depende de los resultados, no ya en tanto fabricación de nuevos productos técnicos, o solución de problemas cotidianos, sino en tanto conclusiones de los procesos experimentales que llegan a confirmar lo predicho por la hipótesis (la cual busca expresar una regularidad natural)

"El experimento no es contemplado en su concordancia con la naturaleza, sino con lo que es planteado en la teoría, y esto planteado en la teoría es el proyecto de la naturaleza según el representar científico-natural" (Heidegger, 2007b:52)

Dado que la concepción de verdad en la ciencia aparece asociada a la idea de resultado y de concordancia con lo mostrado por el experimento, puede aparecer en el ámbito de las ciencias ónticas algo así como la noción de modelo. Un modelo es una estructura teórica que ofrece una versión general de carácter hipotético de un fenómeno de difícil (o imposible) observación. El modelo asegura que, aunque el fenómeno no pueda ser observado o estudiado acabadamente, las mediciones y predicciones podrán seguir siendo realizadas de manera eficiente. Heidegger lo explica de este modo:

"El modelo entra en juego ahí donde los objetos se vuelven invisibles, y donde a pesar de ello, se sigue manteniendo la necesidad de la calculabilidad" (Heidegger, 2007b:286)

Algo así como un modelo teórico sólo puede existir allí donde la referencia directa a los objetos empíricos se muestra como un detalle prescindible. El modelo no busca concordancia con la cosa, sino ser capaz de explicarla satisfactoriamente, y, fundamentalmente poder predecir comportamientos de la realidad estudiada. La existencia de modelos en las ciencias confirma la idea de que la noción de concordancia se ha trastocado a partir de las investigaciones modernas. La adecuación con la realidad es prescindible, pero no puede abandonarse 
la concordancia de las predicciones de la teoría con los resultados del experimento, aún si eso implica pagar el precio de suspender la observación empírica del fenómeno estudiado.

Una última y fundamental conversión que se ha producido en las ciencias ónticas con respecto a la idea de verdad es, tal como Heidegger la denomina en Los seminarios de Zollikon, la transformación de verdad en certeza (Heidegger, 2007b:144). La certeza aparece aquí como el sentido profundo de la verdad, y en definitiva como una de las derivaciones más relevantes de la idea de fundamento. Heidegger nos indica en su lección Principios metafísicos de la lógica, que la certeza nombraría el "estar seguro" de la verdad de un enunciado, la confianza en que hay un fundamento para esta determinada afirmación, luego, la certeza presupone la verdad, y esta es precisamente, una de las formas de enunciar el principio de fundamento o de razón suficiente (Cfr. Heidegger, 2007a:142). La verdad solicita un fundamento para el conocer, una ratio cognoscendi, el terreno de este tipo de fundamentación es el argumento. La certeza, en cambio hace referencia a un estar seguro de la verdad del enunciado, estar cierto de su posesión. Como puede observarse, lo que le da su legitimidad a la posesión de la verdad (certeza) es la intuición de que lo poseído es verdad, es decir, que está efectivamente fundado, por tanto, puede concluirse que el fundamento de la certeza presupone el fundamento de la verdad, o más precisamente, ambos son una y la misma cosa. La noción que surge del análisis de la conexión entre verdad y certeza es que el fundamento de ambas coincide.

"El fundamento de la certeza y el fundamento de la verdad son materialmente lo mismo y sólo se distinguen formalmente mediante el momento de ser conocido y aprehendido" (Heidegger, 2007a:142 -las cursivas son nuestras-).

Ahora bien, es importante destacar que la noción de verdad y certeza no es única y universal para todas las actividades en las que el Dasein se ocupa temático-teoréticamente del mundo; por el contrario, los parámetros de lo que se comprende por verdad, y lo que se solicita para el "estar seguro" de la verdad de una proposición (certeza) varía en cada caso dependiendo del modo en que queden proyectadas de antemano las tareas fácticas de la investigación y la región sobre la que ella se despliega, región que queda definida por una serie de conceptos fundamentales [Grundbegriffe] que funcionan como hilos conductores para el acceso y tránsito del ámbito de estudio.

"Con la elaboración de los conceptos fundamentales de la conductora comprensión del ser se determinan los hilos conductores de los métodos, la estructura del aparato conceptual, la correspondiente posibilidad de verdad y certeza, el modo de fundamentación y demostración, la modalidad del carácter vinculativo y el modo de la comunicación. El conjunto de estos momentos constituye el concepto existencial plenario de la ciencia". (Heidegger, 2006:378)

Realizaremos una aclaración final acerca de la idea de verdad en las ciencias ónticas de lo intramundano: Según la así llamada Noción existencial de la ciencia ${ }^{7}$ [existenziale Begriff der Wissenschaft] -elaborada por Heidegger a lo largo del período de Marburgo, pero expuesta deta-

7 Que aparece en oposición al así llamado "concepto lógico" de la ciencia, al cual nos referimos al comienzo de nuestro estudio. 
lladamente en el parágrafo 69 de Ser y Tiempo- la ciencia constituye un comportamiento que desarrolla explícitamente la comprensión del ser inherente a todo modo del estar-en-el-mundo. Por su parte, la comprensión como existenciario, es mostrada como caracterizada por una posibilidad de explicitación: la interpretación; esta última se realiza desde un horizonte de sentido compuesto de tres estructuras previas: el ver previo, el concebir previo, y el haber previo. Pues bien, el hecho de que toda comprensión se realice siempre desde lo ya tenido - de alguna manera- de antemano, le da a aquella un carácter circular. Las ciencias naturales (en general, las ciencias más influenciadas por el proyecto matemático), y en ciertas versiones, también las ciencias del espíritu, intentarán eliminar toda circularidad de sus conclusiones, sin embargo, en este movimiento, las ciencias realizan una evaluación errónea de la comprensión: el carácter circular no es una falencia que deba ser evitada, sino la estructura misma del comprender. En las ciencias ónticas esta circularidad se observa fundamentalmente en la influencia que los tres niveles de la precomprensión tienen sobre las observaciones empíricas y el desarrollo de teorías. En efecto, toda teoría se desarrolla ante el horizonte de un conjunto de entes preexistentes, una conceptualidad por medio de la cual se habla de las cosas, y un modo específico de observar y acceder a ellas. En general, estos tres modos de aproximación vienen definidos por el proyecto de la comprensión del ser que se encuentra en el origen de toda ciencia ${ }^{8}$. El desafío no será el de escapar a la circularidad, sino ingresar en ella desde una postura crítica, que sea consciente del auténtico origen del modo de ver, concebir y tener ${ }^{9}$. Esta situación nos coloca ante un panorama donde la evidencia, el método, el modo de acceso, y, desde luego, los criterios de verdad resultan diferentes para cada ciencia, e incluso diferentes para la misma ciencia en diversas épocas. La idea de rigurosidad (si por rigurosidad comprendemos la adecuación al objeto de estudio) no será la misma para las disciplinas que hacen de la naturaleza su objeto de estudio que para aquellas que se dedican al Dasein, por ejemplo, en su dimensión histórica. La verdad en las ciencias, al igual que la comprensión tendrá siempre un carácter contextual.

\section{Análisis de la expresión "hay verdad"}

Acudimos ahora a una cuestión destacable en nuestro intento de caracterizar el sentido de verdad que opera como trasfondo en las ciencias, a saber, la discusión en torno a la posibilidad de la subsistencia de la verdad con independencia del despliegue de la existencia.

Ciertamente, suele decirse que "hay" verdad, con anterioridad a la existencia, pero ¿en qué sentido "hay" verdad antes del Dasein? La pregunta presupone una cierta caracterización de la verdad: ésta es comprendida como una propiedad del ente o de los estados de cosas que acontecen ante los ojos. Esta interpretación de la verdad, en tanto independiente del descubrimiento, se asocia con el trascendental verum de la escolástica. Según esta visión tradicional, la verdad sería convertible con el ente, o más bien, todo ente, por el hecho de ser, sería verdadero; también se relaciona con el segundo sentido de verdad expuesto por Heidegger en Lógica, la pregunta por la verdad: es en este sentido que decimos que " 2 × $2=4$ " es "una verdad" o que "toda acción experimenta una reacción de igual intensidad y sentido opuesto" es "una verdad". Ambas proposiciones parecen ser verdaderas, o más precisamente, "verdades" y serlo atemporalmente, y para todo cognoscente. En definitiva, esto es lo que se dice al afirmar que "hay" verdad antes

8 Al igual que la idea de certeza, tal como pudimos observar hace un momento.

9 Para una exposición específica de esta cuestión, remitimos especialmente al parágrafo 32 de Ser y Tiempo. 
del Dasein. Desde esta postura, una verdad sería algo ya constituido en su valor, algo que está allí para nosotros, algo ya capaz de expresar el mundo tal como este se muestra, que contiene una porción efectiva de la realidad, y fundamentalmente, algo que espera ser descubierto.

Pero Heidegger nos advierte a lo largo de sus lecciones del período de Marburgo que lejos de ser algo que aguarda su descubrimiento, la verdad es el proceso mismo de descubrir, y paralelamente, lo que queda descubierto en él. ¿Encontrará entonces la verdad su morada en las proposiciones, o en las cosas?, respondemos: en ambas, pero nunca originariamente. Esta noción se explica en Los problemas fundamentales de la fenomenología:

“La proposición «dos por dos igual a cuatro», en tanto que proposición enunciativa verdadera, es solo verdadera mientras exista el Dasein. Si en principio ya no existe ningún Dasein, ya no vale la proposición, no porque la proposición como tal pierda toda validez, no porque se vuelva falsa y «dos por dos igual a cuatro» se cambie en «dos por dos igual a cinco», sino porque el que algo esté descubierto como verdad sólo puede existir con el Dasein que existe y que descubre." (Heidegger, 2000:270)

La verdad no vive originariamente en el enunciado apofántico; tanto la verdad de la proposición como el carácter de descubierto del ente son posibilitados por la estructura hermenéutica de la existencia, el comprender e interpretar algo en tanto que algo (estructura Als). En su sentido más primario, la verdad no habita, sino que es un hábitat. En un interesante pasaje de Ser y Tiempo se afirma lo siguiente:

"Antes que las leyes de Newton fueran descubiertas no eran "verdaderas"; de lo cual no se sigue que fueran "falsas", ni mucho menos que se volverían falsas si ya no fuera ónticamente posible ningún estar al descubierto" (Heidegger, 2006:247).

Cuando se afirma que las leyes de Newton no eran verdaderas, ¿se quiere decir acaso, que hasta antes de ser formuladas las leyes del movimiento, el mundo no se regía por los principios de inercia, acción-reacción, y semejantes? De ningún modo. Se vivía en una comprensión preconceptual de tales leyes, se habitaba en un mundo que se comportaba de manera legal; en términos de Husserl, experimentábamos el "estilo causal y empírico" del mundo. La afirmación acerca de las leyes de Newton pone de manifiesto el lugar donde radica una confusión fundamental, a saber, la falsa equivalencia entre verdad [Warheit] y efectividad [Wirklichkeit]

Heidegger se ocupa largamente del problema de la efectividad en su curso dictado en Marburgo durante el semestre de verano de 1927, lecciones que aparecen recogidas en la obra Los problemas fundamentales de la fenomenología. En esta obra se ofrece una aguda inspección de la genealogía del concepto de efectividad. Una descripción minuciosa del desarrollo de las reflexiones de aquel curso excede el objetivo de nuestro trabajo, sin embargo, nos serán útiles las siguientes conclusiones: La efectividad nombra el aspecto permanente del ente, el aspecto capaz de persistir por sí mismo, el darse “de hecho”. Según Heidegger, el carácter de persistencia que determina la efectividad, la vuelve otro nombre para la substancialidad, siendo substantia nada menos que la traducción latina de tó hypokeímenon, "lo que subyace", pero también de ousía, interpretado por Heidegger como "lo que está presente". Heidegger propone que ambas nociones (efectividadsubstancialidad) surgen de una interpretación antigua (griega) del ser que toma como hilo con- 
ductor al modelo de la producción de entes artefactuales. En efecto, tanto éidos como idéa, hacen referencia a la metáfora visual (verbo 'oráo), la quidditas, el qué de una cosa está definido por lo visto (idéa) de antemano en la instancia de producción. La figura, y con ella, las notas distintivas de lo que ha de ser producido es lo que guía su confección, es decir que en ellas está contenido el qué de lo fabricado, tó tí én eínai, "lo que era el ser" para esa cosa, literalmente, en latín, su essentia.

Ahora bien, dado que estos conceptos surgen del modelo de la producción, lo así definido quedará determinado por las notas propias del ente artefactual: fundamentalmente, la independencia de la instancia productora, la separación de la causa eficiente, la posesión de un ser propio, y no subordinado a otro. ${ }^{10}$ Por lo dicho se ve que la efectividad nombra una independencia, una subsistencia, una capacidad de ser en-sí, una cierta disposición a mantenerse por sí mismo, con olvido de la fase productora que funciona como fundamento de ser. Esto es lo que afirmamos cuando hablamos de efectividad.

Con la noción de efectividad (subsistencia) delimitada, podemos aclarar por qué este concepto y el de verdad no deben ser confundidos, ni tomados como sinónimos. En el lenguaje heideggeriano de Ser y Tiempo ${ }^{11}$, la noción de efectividad encuentra un término paralelo, se trata de la Vorhandenheit, dicho de un ente, la condición de ser-ahí ante los ojos. En la analítica existencial, este concepto habla de la independencia de un ente con respecto al interés productivo del Dasein, independencia que perfila al ente en su aspecto "objetivo", dotado de notas esenciales que le son propias. Sin embargo, y sin dudas, "El paso de Zuhandenheit a Vorhandenheit, el cual se plasma en la ciencia, sólo es posible como una determinada forma del interés" (Tugendhat, 1970:295) El interés del Dasein que se expande sobre el mundo no queda suspendido, sino que sólo modifica su estilo pragmático en uno teórico. El ente perfilado por esta conversión sugiere una permanencia más allá de los comportamientos con los que una existencia se refiera a él. Entonces, ¿dónde radica la confusión entre efectividad y verdad?

En la opinión cotidiana, se admite, como uno de los sentidos posibles, que la verdad constituye una propiedad de los hechos y objetos, a la cual el discurso debe someterse; el conocimiento debe ocuparse de acceder a aquellos objetos y fenómenos, y hacerlos manifiestos mediante la expresión enunciativa. Pareciera que mientras el conocimiento no triunfa en su labor manifestante, los objetos y hechos no pierden su carácter de verdad, puesto que seguirían teniendo una influencia en el mundo, una cierta independencia, verificable a posteriori. Así, podría comprobarse ulteriormente que mientras estos entes y fenómenos permanecieron ocultos, ningún aspecto de su "esencia" se vio modificado, y su operar sobre el mundo nunca resultó interrumpido. Desde esta perspectiva, el hecho de no haber descubierto aún aquellos fenómenos y estados de cosas, no suspendería su influjo sobre el mundo, su subsistencia, y por tanto, no disminuiría su verdad. Cuando tales leyes, entes y hechos son desocultados por la investigación científica, advendría un consiguiente proceso de acreditación retrospectiva: la confirmación de que esta ley hoy expresada en proposiciones, rige actualmente, y siempre ha regido el estilo causal del mundo. En otras palabras, según esta postura, la expresión en proposiciones jamás vuelve verdadera una ley, simplemente expresa la verdad que ella siempre ha contenido.

10 A menos, por supuesto, que se piense a Dios como productor supremo, y responsable de todo el ens creatum. Sin embargo esta interpretación se aleja del originario sentido griego.

11 Y también en las lecciones de Marburgo que funcionan como la "nueva elaboración de la tercera sección". 
Pero -y aquí yace la distinción- ejercer un influjo sobre el mundo, participar en un encadenamiento causal, introducir cambios en los estados de cosas, regir las relaciones entre entes, o el simple y llano subsistir con independencia del momento productor, no son caracteres convertibles con el ser verdadero de una ley.

En este punto se vuelve necesaria una aclaración: una ley científica -como las que ahora consideramos- constituye el resultado de una actitud muy específica por medio de la cual la existencia se relaciona con el mundo, nos referimos a la actitud teorética de la ciencia ${ }^{12}$. Las regularidades descubiertas por esta actitud serán expresadas en proposiciones o enunciados apofánticos -el tipo de enunciado que es susceptible de ser calificado como verdadero o falso-. De hecho, según el así llamado concepto lógico de ciencia (Cf. Heidegger, 2009:16 y 2006:373), los discursos teóricos consistirían precisamente en amplios entramados de proposiciones. Heidegger afirma una y otra vez a lo largo del período de Marburgo que la verdad de una ley se funda en la verdad antepredicativa del llano ser-en-el-mundo. Ahora bien, es necesario distinguir la verdad como ser descubridor [Entdeckend sein] (inherente al despliegue aperiente de la existencia), la verdad como estado de descubierto [Entdeckheit] (que corresponde al ente o fenómeno que queda desocultado por aquel despliegue), y la verdad como calificativo de una proposición (que se refiere a la concordancia de una proposición mostrativa con un estado de cosas mundano). El sentido en el cual se llama verdadera a una ley científica, es, como hemos visto, el tercero.

La verdad de una ley científica pertenece a un género diferente del de la efectividad; las propiedades y disposiciones de los estados de cosas se muestran ante una aproximación fenomenológico-hermenéutica como aquello que no posee sentido de suyo, sino hasta tanto resulta incorporado por la existencia que descubre. Ante esta situación, podría surgir el interrogante acerca de la caracterización de aquella área de fenómenos y entes donde la existencia aún no ha desplegado su impronta comprensora. Para orientarnos en esta búsqueda, y aclarar la distinción entre la verdad de la ley científica y la efectividad del darse de los estados de cosas, podríamos encontrar un valioso aporte en la semiótica de Iuri Lotman (Lotman, 2000), específicamente, en lo referente al área por él denominada "lo alosemiótico".

A lo largo de diversos artículos, Lotman desarrolla la noción de semiosfera, la cual mienta, en sus propias palabras, “...el gran sistema...fuera del cual es imposible la existencia misma de la semiosis” (Lotman, 2000:23). El concepto de semiosfera nombra el ámbito máximo de interrelación semántica de donde proviene y hacia donde se reconduce todo sentido. Si seguimos la interpretación de Bertorello (2005), el concepto de semiosfera podría relacionarse eficazmente con la noción heideggeriana de mundo; en efecto, ambos conceptos hacen referencia a aquel contexto de producción de sentido en el cual redunda toda comprensión. El sentido, en tanto horizonte de comprensibilidad de los entes -horizonte siempre social e históricamente constituido-, surge del despliegue del interés humano sobre una región de interacción. Dado que el mundo puede interpretarse como un entramado de relaciones pragmáticas que poseen a la existencia como el centro hacia el que se dirigen todas las condiciones respectivas, el mismo se nos muestra como un entretejido de significados, es decir, un texto. Tanto en la semiótica de Lotman, como en la fenomenología hermenéutica de Heidegger, el mundo ostenta un carácter textual que se vuelve apreciable en su dimensión relacional-significativa, esto es, la significatividad (Bedeutsamkeit) (Cf. Heidegger, 2006:§18)

12 Para un tratamiento más exhaustivo de la actividad científica como actitud o modalidad derivada de la relación con el mundo, (noción existencial de la ciencia) remitirse a Heidegger 2006: §69b 
De lo dicho se extrae el marco pertinente para responder a la pregunta: ¿a qué espacio pertenece todo aquello que no ha sido aún alcanzado por la comprensión del proyectar humano? Consideramos que, efectivamente, existe un ámbito para todo lo que, si bien no ha sido descubierto por el interés de la existencia, no puede afirmarse que sea una nada. Según Lotman, hay una esfera que se ubica más allá del espacio semántico; esta región es denominada lo alosemiótico o extrasemiótico. Estrictamente, lo alosemiótico constituye un espacio hipotético, puesto que, por definición, aún no ha sido abarcado por semiosis alguna y por tanto, no puede ser objeto de experiencia. Sin embargo, este mismo carácter misterioso es el que reviste a un fenómeno que todavía es ajeno al proyectarse del Dasein. Consideramos que la noción de lo alosemiótico podría ofrecer un nombre para esa región integrada por todo aquello que aún no ha sido alcanzado por la comprensión descubridora. Esto nos coloca ante un panorama donde se observan diversos modos de la verdad y el descubrimiento, un cierto escalonamiento en el que las formas más básicas fundan a las más derivadas. Así podríamos mencionar: a) la verdad como existenciario (apertura), esto es, la condición de posibilidad para el antepredicativo descubrir, inherente a toda existencia, donde rige el prelógico mostrarse del ente; b) la verdad como el ejercicio fáctico del descubrimiento, o dicho de otro modo, el ser descubridor de la existencia, en tanto proyecto que, al derramar su interés sobre el mundo, pone en libertad a los entes en vistas a su sentido; c) el ser verdadero de aquello que es puesto en libertad por el descubrir; y d) la verdad como calificativo del enunciado apofántico, componente estructural de los discursos teóricos.

Frente a esta descripción, comprendemos que los entes y estados de cosas deben, en cierto modo, presuponerse a los diversos modos del discurrir enunciativamente acerca de ellos. En sus lecciones sobre El Sofista de Platón, Heidegger indica que el aprehender consiste en un "dejar que algo sea visto por el lógos" (1992a:183) Esta idea encuentra un paralelo en la siguiente expresión de Lotman:

"El «carácter cerrado» de la semiosfera se manifiesta en que ésta no puede estar en contacto con los textos alosemióticos o con los no-textos. Para que éstos adquieran realidad para ella, le es indispensable traducirlos a uno de los lenguajes de su espacio interno o semiotizar los hechos no-semióticos"(Lotman, 2000:12) ${ }^{13}$

Sin embargo, en una obra posterior, Heidegger, de manera aparentemente contrastante, indica que estamos "dominados" por el ente (Cfr. 2001a:142) Estas dos afirmaciones (que señalan, respectivamente, una preeminencia del ente y del discurso) no se contraponen en la fenomenología hermenéutica, dado que en este sistema la estructura de la comprensión tiene un carácter circular. Sugerimos que las dos propuestas heideggerianas podrían estar describiendo nada menos que el ingreso a la semiosfera, un cierto antecedente a la teoría de Lotman.

Retomando la discusión en torno a la verdad/efectividad de las leyes científicas, consideramos que si centramos el análisis únicamente en las leyes de Newton, podría resultar un tanto arduo determinar la diferencia entre el estado descubierto de una regularidad y un estado previo a todo descubrimiento -puesto que las tres leyes del movimiento resultan experimentables cotidianamente, aunque aún no se encuentren enunciadas por medio de una teoría- En cambio,

13 Desde luego, cabe realizar una importante salvedad: el Mundo de Heidegger no posee este carácter "cerrado" de la semiosfera, sino todo lo contrario, un carácter abierto. El mundo de la fenomenología hermenéutica no es en modo alguno una cápsula. 
tal vez, si nos concentrásemos en otros fenómenos de observación más difícil (o imposible), la diferencia entre efectividad y verdad podría volverse más clara. Pensemos por ejemplo en el proceso de mitosis celular, o la transición hiperfina del hidrógeno. En estos casos, la existencia no posee una experiencia cotidiana y antepredicativa de los procesos -como ocurría en la experiencia del comportamiento inercial y gravitacional del mundo de la vida- La comprensión primaria simplemente no accede a estos fenómenos a menos que realice un movimiento de desmundanización tematizante, que casi siempre implica el ingreso a la actitud teórica de la ciencia. En estos nuevos casos que sugerimos, ni si quiera el prelógico mostrarse del ente habría caído bajo el espectro del interés de la existencia; sin embargo, no por ello podríamos afirmar que tales fenómenos no tenían ya lugar en la naturaleza ${ }^{14}$.

En el caso de las leyes de Newton, los procesos se encontraban en un primario y habitual estado de desoculto, a saber, la existencia convivía con el antepredicativo darse de aquellos fenómenos, y, únicamente en este sentido se podría afirmar que los procesos eran "verdaderos". Sin embargo, un proceso no es lo mismo que una ley; en efecto, la ley enuncia teóricamente una regularidad observada en un proceso.

Pues bien, ¿qué ocurre con aquellos fenómenos que aún no han sido enunciados por medio de leyes o bien, aún no han sido si quiera desocultados por el interés aperiente de la existencia? Los estados de cosas sobre los cuales la existencia no se ha desplegado quedan confinados al ámbito de lo aún ajeno a la semiosis, y por lo mismo, ajeno a la comprensión fáctica. Lo alosemiótico nombraría aquel ámbito integrado por la totalidad de los entes y fenómenos que no han sido alcanzados por la apertura de la existencia, pero que no por ello dejan de ejercer una influencia en el mundo (efectividad). El concepto de Lotman se nos muestra como una herramienta para pensar el ingreso de un estado de cosas, primero al comprender primario y antepredicativo, y luego, al universo teórico de la ciencia.

En efecto, el sentido -o la semiosis, en términos de Lotman-sólo acontece en la esfera del interés del Dasein, y así lo afirma claramente Heidegger:

"Sólo el Dasein "tiene" sentido, en la medida en que la aperturidad del estar-en-elmundo puede ser "llenada" por el ente en ella descubrible. Por eso, sólo el Dasein puede estar dotado de sentido o desprovisto de él” (Heidegger, 2006: 175)

Ahora bien, cuando se afirma que las leyes de Newton no eran verdaderas, no se pretende sostener que ellas no regían efectivamente los movimientos de los entes, sino simplemente que carecían del estado de desoculto propio de un ente manifestado por la aperturidad. Un análisis convergente con los de Ser y Tiempo y Lógica, la pregunta por la verdad se realiza en Los problemas fundamentales de la fenomenología. Allí se afirma:

“Antes de su descubrimiento, las leyes de Newton no eran ni verdaderas ni falsas. Esto no puede querer decir que el ente que se descubre con las leyes develadas no haya sido antes tal como se mostró después del descubrimiento ni que sea tal como se muestra. El estar-descubierto, o sea, la verdad, devela el ente precisamente como lo que ya era antes, independiente de su estar o no descubierto". (Heidegger, 2000:269-270)

14 Entendemos aquí el término naturaleza como "el conjunto exterior de lo que está ahí ante los ojos (vorhandenheit)" 
En este punto se vuelve relevante la noción de manifestabilidad pre-lógica [vorlogische Offenbarkeit], concepto que nombra el modo sintético de aparecer del ente, la unidad originaria del ente y sus determinaciones; esta manifestabilidad antepredicativa puede ser captada en un acto de aprehensión simple donde el ente y sus perfiles aparecen reunidos, ej. la intuición "pizarra negra"15; sin embargo, si bien es cierto que el descubrir -el cual, posteriormente puede desarrollarse en el enunciar que, por medio de un realce, establece al mismo tiempo una síntesis y una diáiresis del ente y sus determinaciones- se pliega a aquel previo aparecer como unido, este modo pre-lógico de venir a la presencia no constituye por sí mismo una verdad, más bien se trata del correlato del movimiento desocultante de la existencia. De hecho, tal como observamos hace un momento, Heidegger indica que la aprehensión simple del comparecer del ente como fenómeno unitario no encuentra en él una verdad que aquel ente poseía como propiedad, sino que sólo "permite que algo sea visto por el lógos" esto implica que no hay apertura y descubrimiento (verdad) que se encuentre por fuera del despliegue de las estructuras existenciarias del Dasein.

Estrictamente, la estructura sintética del ente a la que se adapta la predicación no es verdadera de suyo $^{16}$, sólo hablaremos de verdad cuando el Dasein haya desplegado sus posibilidades de existencia sobre los entes del mundo, ya sea acompañando el modo de aparecer, o defraudándolo en un "disimular" (Cfr. Tugendhat 1970:333)

Al afirmar que las leyes de Newton no eran verdaderas, se trata de indicar que aún no habían sido mostradas en lo que eran, en el cómo de su regir, y, desde luego, que aún no habían sido incluidas en un proyecto de la constitución de ser, ni formaban parte de una región ontológica; por ello, aún no podían ser tematizadas en proposiciones, y consecuentemente, tampoco les era permitido el ingreso al complejo de proposiciones que conforma la ciencia (en sentido lógico).

Ahora bien, el Dasein nunca comprende desde una absoluta falta de noticia ${ }^{17}$, es decir que antes de su formulación, vivíamos inmersos en las leyes, participábamos de un comprender previo, nuestros proyectos fácticos se desarrollaban en el interior de una vivencia habitual del modo inercial y gravitacional del acontecer mundano; sin embargo, hizo falta la llegada de un Dasein descubridor para introducir en aquellos patrones una modificación de estatus, que los calificó de verdaderos, en el sentido de desocultos. Esta concepción se observa claramente enunciada en Los problemas fundamentales de la fenomenología: "Para que la naturaleza sea como es, no se precisa de la verdad, es decir, del estar descubierto" (Heidegger, 2000:270). Para el caso particular de las ciencias, incluso antes de este descubrimiento debió acontecer una apertura previa de una región ontológica y una demarcación (ingenua y general en principio) ${ }^{18}$ de los caracteres de ser de los entes de esta región. Sólo desde esta apertura previa, y sólo ante el horizonte de un modo predeterminado de permitir el comparecer del ente, pudo un Dasein descubridor realizar la apertura de las regularidades que rigen los fenómenos, y enunciarlas en proposiciones.

15 Estrictamente, esta intuición no necesita estar asociada a una expresión verbal.

16 En casos de este tipo, hablaremos de verdad únicamente en la medida en que semejante manifestabilidad haya sido puesta en libertad por un Dasein descubridor. En otras palabras, el sentido de verdad que se aplica al ente, es el de estado de descubierto.

17 Naturaleza del círculo hermenéutico

18 Cfr. Heidegger, 2006:32 
Diremos aún más, si la ley es un tipo de proposición, cuyo contenido ideal es una regularidad mundana, y ya interpretamos la formulación de proposiciones como una posibilidad del cuidado, fundada en el primario descubrir de la apertura, puede concluirse que, mientras no fueron descubiertas, las leyes de Newton sencillamente no eran leyes. Podría calificarse a aquellas regularidades vivenciales de "efectivas", "fácticas", "experienciales", "operantes", pero no aún de "verdaderas", ni tampoco "legales". En virtud de las leyes de Newton, ciertos entes y fenómenos se hicieron accesibles para el Dasein. Así es como opera la verdad, el ente se muestra como este que ya era antes, adquiriendo la configuración formal de "esto es ello mismo", pero nunca desde una absoluta incomprensión.

De lo dicho se desprende que la verdad es un constitutivo de la estructura hermenéutica de la existencia; de la aperturidad en tanto sentido del Ahí. Decir que "hay" verdad es afirmar que el Dasein es esencialmente descubridor:

"que hay "verdades eternas" sólo quedará suficientemente demostrado cuando se logre probar que el Dasein fue y será por toda la eternidad. Mientras esto no se pruebe, la frase no dejará de ser una afirmación fantástica que no cobra legitimidad por el hecho de que ordinariamente es "creída" por los filósofos" (Heidegger, 2006: 247)

Todas las reflexiones precedentes, y el estudio del modo de consideración de una ley de la física, nos llevan ahora a confirmar una situación ya señalada: el sentido de verdad que funciona como trasfondo para las actividades de las ciencias ónticas es el de la verdad como calificativo de la proposición, o en otras palabras, la idea de que el lugar de la verdad es la proposición enunciativa (apofántica); la opinión que Cristoph Martel denominó "Tesis del lugar" [Ortsthese] (Cfr. Martel, 2008:45). Las investigaciones científicas naturales coinciden en no considerar al ente para el cual la verdad es un comportamiento esencial; ellas no son capaces de detectar (ni se lo proponen) la implicancia de la existencia en el descubrimiento de los entes y regularidades; ellas no se preguntan por el ente para el cual la naturaleza comparece como regular y determinable cuantitativamente.

La discutida posibilidad de "verdades eternas" se desarma cuando consideramos que la verdad constituye originariamente un carácter de ser del Dasein, de su comportamiento hacia el mundo. La imposibilidad de proponer un Dasein que exista eternamente, y, por tanto, el rechazo de la idea de verdades "atemporales", y la consiguiente afirmación de que sólo hay verdad mientras el Dasein existe, no nos arroja en el territorio del relativismo o el escepticismo. Estas dos posturas filosóficas surgen en lucha con una concepción errónea de la verdad, que la vuelve un factor atemporal, una determinación del sujeto, objeto, o del sentido. La propuesta heideggeriana no postula la imposibilidad de la verdad, sino que la circunscribe a su correcto ámbito: el despliegue de la existencia; el planteo de Heidegger simplemente construye una noción "deflacionada" de la verdad (a los ojos de las posturas tradicionales y realistas). Para finalizar, escuchemos a Heidegger exponer estas últimas nociones claramente:

"Proponer verdades eternas sigue siendo una afirmación fantástica de la misma forma que es una tergiversación ingenua creer que, si sólo hay verdad mientras exista el Dasein, estamos abocados al relativismo y al escepticismo. Por el contrario, las teorías del relativismo y del escepticismo surgen a partir de una oposición, en parte justificada, a un absolutismo y un dogmatismo erróneos del concepto de verdad que 
tienen su fundamento en que se toma el fenómeno de la verdad superficialmente como determinación del sujeto o del objeto o, incluso, si no valen estos, como determinación de un tercer ámbito, el del sentido" (Heidegger, 2000:271)

La visión existencial sólo indica que la verdad no es originariamente una propiedad de los sujetos ni de los objetos. Esta concepción no equivale al escepticismo o relativismo -para los cuales la verdad resulta imposible o inaccesible- puesto que ambos planteos trabajan con nociones de verdad que difieren de la heideggeriana. Escepticismo y relativismo se enfrentan a concepciones erradas (dogmáticas y absolutistas) de la verdad, la concepción de Heidegger dista mucho de esta caracterización.

\section{Conclusión}

A modo de conclusión, y según lo propuesto en la introducción, reconstruimos los sentidos en los que se emplea el término "verdad" en el ámbito de las ciencias ónticas de lo intramundano, tal como lo ha mostrado nuestro recorrido de las más pertinentes obras del período de Marburgo:

a) La interpretación más difundida de la noción de verdad dentro de la esfera de las ciencias es aquella que hace de ella un carácter de la proposición (enunciado apofántico). Esta lectura considera que el enunciado mostrativo es el lugar propio de la verdad. El desarrollo y ampliación de esta postura es el que da lugar al surgimiento del así llamado "concepto lógico" de la ciencia, es decir, la interpretación que considera a la ciencia como un entramado de enunciados apofánticos que se vinculan por relaciones de fundamentación. El enunciado (lugar de la verdad) sería la unidad mínima de análisis de las ciencias, y, por tanto, el armazón completo de una disciplina teórica quedaría estructurado en torno a este elemento básico y esencial ${ }^{19}$

b) En segundo lugar, la verdad es entendida como resultados; noción que puede ser interpretada de dos modos: una de ellas emana de la experiencia de la cotidianidad media, y la otra, de las tareas específicas del ambiente científico. Para la medianía, para el "hombre de la calle", la verdad de la ciencia se ve reflejada en los resultados de las investigaciones, los cuales quedan plasmados en los artefactos, procedimientos y técnicas que se despliegan sobre en mundo socialmente compartido, y benefician (o perjudican) a los individuos. El hombre común se deja beneficiar o se ve afectado por los resultados de las teorías, y presupone (confía) que si estos resultados han llegado a formar parte del mundo social, debe existir una teoría que los sustenta, un conjunto de verdades que le dan explicación y origen. Ahora bien, diferente es la situación del hombre de ciencias; los especialistas también suelen asociar la veracidad de una teoría a los resultados que ella produce, pero no ya en el sentido de principios y leyes que quedan plasmados en artefactos o técnicas, sino en el de resultados que emanan de un experimento. En efecto, en el ámbito de la ciencia se suele considerar que una teoría gana en veracidad si ella resulta confirmada por el resultado del experimento diseñado para someterla a prueba. Esta consideración nos introduce en un tercer sentido de verdad en las ciencias:

c) La tercera lectura interpreta la verdad como adecuación, pero no en el sentido escolástico de adaequatio intellectus et rei, sino como la concordancia entre la predicción hecha por la teoría y el resultado del experimento que la pone a prueba. Una hipótesis (elemento central

19 por extensión, también se incluyen en este armazón instituciones, equipamientos, especialistas, etc. 
de una teoría) ganará en veracidad, si sus predicciones son confirmadas por el experimento. Desde luego, ninguna teoría puede ser contrastada con todos los casos posibles del fenómeno que ella busca explicar; la experimentación es incapaz de llevarnos ante lo universal, sin embargo, las teorías se expresan en términos universales (incluso aquellas que responden a un esquema probabilístico de resultados, ya que ninguna ley se elabora para regir un caso singular). Dado que la ley se expresa en términos universales a pesar de que la totalidad de los casos posibles resulta inaccesible, Heidegger describe las labores de las ciencias de lo intramundano como la construcción de una ficción (Cfr. Heidegger, 2007b:184).

d) Por último, pudimos ver que la ciencia ha producido la así llamada "conversión de la verdad en certeza", esto es, la transformación de la adecuación de las proposiciones con el fenómeno por ella descriptas, en la confianza en que existe un fundamento para cada afirmación, esto es, una base explicativa compuesta por el conjunto de razones para el funcionamiento de los procesos observados. Un fenómeno, descripto por medio de una serie de enunciados apofánticos de carácter teórico deberá contar con fundamentos explicativos, proposiciones más básicas y anteriores que no necesitan ser explicitadas con ocasión de cada nueva afirmación teorética. Este linaje de proposiciones cada vez más básicas se remonta hasta una serie de enunciados que no son susceptibles de demostración por medio de premisas anteriores, sino que sólo pueden ser objeto de una acceptio. (Cfr. Heidegger, 2007b:27)

\section{Bibliografía}

Bertorello, A. (2005b) “Semiosfera y Mundo: ensayo sobre un posible diálogo entre Lotman y Heidegger", en Revista LSD: Lenguaje, Sujeto y Discurso, №1, Buenos Aires, pp. 15-19

Guignon, CH. (1983): Heidegger and the problem of knowledge, Indiana, USA: Hackett publishing company

Heidegger, M. (1992). Platon: Sophistes, Frankfurt am Main: Vittorio Klostermann

Heidegger, M. (2000): Los problemas fundamentales de la fenomenología, Madrid:Trotta, Trad. y prólogo de Juan José García Norro.

Heidegger, M. (2006) Ser y Tiempo, Madrid: Trotta. Traducción y notas de Jorge Eduardo Rivera

Heidegger, M. (2007a) Principios metafísicos de la lógica, Madrid: Síntesis. Traducción de Juan José García Norro

Heidegger, M. (2007b) Seminarios de Zollikon: protocolos, diálogos, cartas. México: Jitanjáfora $\mathrm{M}^{\mathrm{o}}$ Relia Ed. Trad. De Ángel Xolocotzi Yáñez

Heidegger, M. (2009) Tiempo e Historia, Madrid: Trotta. Edición y traducción de Jesús Adrián Escudero

Husserl, E. (2006) Investigaciones Lógicas, tomo I, Madrid: Alianza, Trad. de Manuel G. Morente y José Gaos.

Kockelmans, J.J. (1985): Heidegger and science, Washington D.C.:Center for advanced research in phenomenology \& University press of America

Lotman, I. (2000) La Semiosfera I ,Semiótica de la cultura y el texto. Valencia: Ed. Cátedra, traducción de Desiderio Navarro

Martel, CH. (2008): Heideggers Warheiten: Warheit, Referenz und Personalität in Sein und Zeit. Berlin-New York: Walter de Gruyter

Tugendhat, E.(1970) Der Warheitsbegriff bei Husserl und Heidegger, Berlin: Walter de Gruyter \& Co. 